Laporte. C., O'Connor, R. and Garcia Paucar, L., The Implementation of ISO/IEC 29110 Software Engineering Standards and Guides in Very Small Entities, In L.A. Maciaszek and J. Filipe (Eds.) Evaluation of Novel Approaches to Software Engineering, Springer Verlag, CCIS 599, pp. 162-179, 2016.

\title{
The Implementation of ISO/IEC 29110 Software Engineering Standards and Guides in Very Small Entities
}

\author{
Claude Y. Laporte ${ }^{1}$, Rory V. O’Connor ${ }^{2}$ and Luis Hernán García Paucar ${ }^{3}$ \\ ${ }^{1}$ École de technologie supérieure, Montréal, Canada \\ ${ }^{2}$ Schoolof Computing, Dublin City University, Dublin, Ireland \\ ${ }^{3}$ Universidad Peruana de Ciencias Aplicadas, Lima, Peru \\ Claude.Y.Laporteletsmtl.ca, roconnorecomputing.dcu.ie, \\ luis.garcia@upc.edu.pe
}

\begin{abstract}
This paper outlines the details of seven case studies involving the pilot usage of the new standard ISO/IEC 29110standard 'Lifecycle Profiles for Very Small Entities', which was specifically designed by Working Group 24 of ISO/IEC JTC1/SC7 to address the standardization needs of Very Small Entities (VSEs). The purpose of this paper is to add substantially to the body of knowledge and the literature on the rollout and implementation of this new and evolving standard and to act as guidance for other researchers in the design and implementation of ISO/IEC 29110 case studies. Furthermore it is hoped that that the lessons learnt from these case studies will help promote the adoption of this new standard in an industrial setting.
\end{abstract}

Keywords. Very Small Entities, ISO Standards, ISO/IEC 29110, VSE.

\section{INTRODUCTION}

In the domain of software development, small and very small companies have the challenge of handling multiple small-scale, fast-moving projects allowing little room for unwieldy management processes, but still requiring an efficient and straightforward monitoring process [1]. Moreover due to the small number of people involved in the project and the organization, most of the management processes are performed through an informal way and less documented [2]. The perception of heavyweight processes, especially in terms of documentation, cost and nonalignment with current development process, are among the reasons why the companies did not plan to adopt a lifecycle standard in the short to medium term $[3,4]$.

The definition of "Small" and "Very Small" Entities is challengingly ambiguous, as there is no commonly accepted definition of the terms. The term "very small entity" (VSE) had been defined by the ISO/IEC JTC1/SC7 Working Group 24 and subsequently adopted for use in the new ISO/IEC 29110 process lifecycle standard as being "an entity (enterprise, organization, department or project) having up to 25 people" [5]. 
Industry recognizes the value of Very Small Entities (VSEs) in contributing valuable products and services. A large majority of enterprises worldwide are VSEs. A large majority of enterprises worldwide are VSEs. For example in Canada, close to 98 percent of businesses are small businesses with fewer than 50 employees. About 32 percent of these have between one and 19 employees [6].

VSEs have unique characteristics, which make their business styles different to larger organizations and therefore most of the management processes are performed through a more informal and less documented manner [7]. Furthermore there is an acknowledged lack of adoption of standards in small and very small companies, as the perception is that they have been developed for large software companies and not with the small organisation in mind $[8,43]$. As smaller software companies have fewer resources in term of people and money there are many challenges [9].

There is evidence that the majority of small and very small software organizations are not adopting [54] existing standards/proven best practice models because they perceive the standards as being developed by large organizations and orientated towards large organizations, thus provoking the debate the in terms of number of employees, size does actually matter $[10,44]$. Studies have shown that small firms' negative perceptions of process model standards are primarily driven by negative views of cost, documentation and bureaucracy [11]. In addition, it has been reported that SMEs find it difficult to relate standards to their business needs and to justify the application of the international standards in their operations [12]. Most SMEs cannot afford the resources for, or see a net benefit in, establishing software processes as defined by current standards and maturity models [13].

Accordingly, a new standard ISO/IEC 29110 "Lifecycle profiles for Very Small Entities" is aimed at meeting the specific needs of VSEs [14]. The overall objective of this new standard is to assist and encourage very small software organizations in assessing and improving their software process and it is predicted that this new standard could encourage and assist small software companies in assessing their software development process [50]. The approach [15] used to develop ISO/IEC 29110 started with the pre-existing international standards, such as the software life cycle standard ISO/IEC/IEEE 12207 [40, 41] and the documentation standard ISO/IEC/IEEE 15289 [42].

The working group behind the development of this standard is advocating the use of pilot projects as a mean to accelerate the adoption and utilization of ISO/IEC 29110 by VSEs [7]. Pilot projects are an important mean of reducing risks and learning more about the organizational and technical issues associated with the deployment of new software engineering practices [16]. To date a series of pilot projects for the software engineering profile standard have been completed in several countries with the results published in a variety of literature $[17,18,19,20]$.

\section{THE ISO/IEC 29110 STANDARD FOR VSES}

The working group (WG24) of the ISO/IEC JTC1 SC7 mandated to develop the new set of standards for VSEs, used the concept of ISO standardized profiles (SP) from 
ISO/IEC/IEEE 12207 to develop the new standards for VSEs developing software. From a practical point of view, a profile is a kind of matrix, which identifies precisely the elements that are taken from existing standards from those that are not. The overall approach followed by WG24 to develop this new standard for VSE consisted of the following steps:

- develop a set of profiles for VSEs not involved in critical software development,

- select the ISO/IEC/IEEE 12207 process subsets applicable to VSEs having up to 25 people,

- select the description of the products, to be produced by a project, using ISO/IEC/IEEE 15289 standard

- develop guidelines, checklists, templates, examples to support the subsets selected.

The basic requirements of a software development process are that it should fit the needs of the project and aid project success [21, 22]. And this need should be informed by the situational context where in the project must operate and therefore, the most suitable software development process is contingent on the context [23, 24]. The core situational characteristic of the entities targeted by ISO/IEC 29110 is size.

Profile Groups are a collection of profiles. The Generic Profile Group has been defined as applicable to VSEs that do not develop critical software. This Profile Group is a collection of four profiles (Entry, Basic, Intermediate, Advanced) providing a roadmap to satisfying a vast majority of VSEs worldwide. VSEs targeted by the Entry Profile are VSEs working on small projects (e.g. at most six person-months effort) and for start-up VSEs. The Basic Profile describes software development practices of a single application by a single project team of a VSE. The Intermediate Profile is targeted at VSEs developing multiple projects with more than one project team. The Advanced Profile is target to VSEs which want to sustain and grow as a competitive software development business.

\subsection{The ISO/IEC 29110 Basic Profile}

At the core the Basic Profile of this standard is a Management and Engineering Guide, officially know as ISO/IEC TR 29110-5-1-2 [52], which focuses on Project Management and Software Implementation as illustrated in Figure 1. The purpose of the Basic Profile is to define Software Implementation (SI) and Project Management (PM) processes from a subset of ISO/IEC/IEEE 12207 and ISO/IEC/IEEE 15289 [42] appropriate for VSEs, as illustrated in Figure 2.

The purpose of the Basic Profile is to define Software Implementation (SI) and Project Management (PM) processes from a subset of ISO/IEC/IEEE 12207 and ISO/IEC/IEEE 15289 appropriate for VSEs. The main reason to include project management is that the core business of VSEs is software development and their financial success depends on successful project completion within schedule and on budget, as well as on making a profit. The high-level view and the relationships between the Software Implementation Process and the Project Management processes are illustrated in Figure 2. 
This standard defines two processes: Software Implementation and Project Management. The purpose of the Software Implementation process is the systematic performance of the analysis, design, construction, integration and tests activities for new or modified software products according to the specified requirements. The purpose of the Project Management process is to establish and carry out in a systematic way the tasks of the software implementation project, which allows complying with the project's objectives in the expected quality, time and cost.

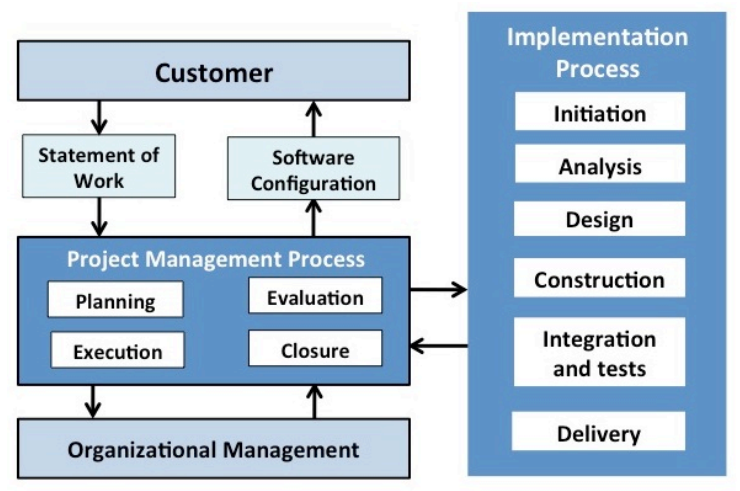

Fig. 1. Basic profile processes and activities [26]

The seven objectives of the PM process are [52]:

1. The Project Plan for the execution of the project is developed according to the Statement of Work and reviewed and accepted by the Customer.

2. Progress of the project is monitored against the Project Plan and recorded in the Progress Status Record.

3. The Change Requests are addressed through their reception and analysis. Changes to software requirements are evaluated for cost, schedule and technical imp

4. Risks are identified as they develop and during the conduct of the project.

5. A software Version Control Strategy is developed. Items of Software Configuration are identified, defined and baselined.

6. Software Quality Assurance is performed to provide assurance that work products and processes comply with the Project Plan and Requirements Specification.

The four activities of the Project Management Process are [52]:

- Project Planning: The primary objective of this process is to produce and communicate effective and workable project plans.

- Project Plan Execution: To implement the actual work tasks of the project in accordance with the project plan.

- Project Assessment and Control: Purpose is to determine the status of the project and ensure that the project performs according to plans and schedules, within projected budgets and it satisfies technical objectives. 
- Project Closure: Typically involves releasing the final deliverables and communicating project closure to all stakeholders.

The purpose of the Software Implementation process is to achieve systematic performance of the analysis, design, construction, integration, and test activities for new or modified software products according to the specified requirements. The seven objectives of the SI process are [52]:

- Tasks of the activities are performed through the accomplishment of the current Project Plan.

- Software requirements are defined, analyzed for correctness and testability, approved by the Customer, baselined and communicated.

- Software architectural and detailed design is developed and baselined. It describes the Software Components and internal and external interfaces of them.

- Software Components defined by the design are produced. Unit test are defined and performed to verify the consistency with requirements and the design.

- Software is produced performing integration of Software Components and verified using Test Cases and Test Procedures. Results are recorded at the Test Report.

- A Software Configuration, that meets the Requirements Specification as agreed to with the Customer, which includes user, operation and maintenance documentations, is integrated, baselined and stored at the Project Repository.

- Verification and Validation Tasks of all required work products are performed using the defined criteria to achieve consistency among output and input products in each activity.

The activities of the Software Implementation Process are [52]:

- Software Implementation Initiation: Ensures that the Project Plan established in Project Planning activity is committed to by the Work Team.

- Software Requirements Analysis: Analyzes the agreed Customer's requirements and establishes the validated project requirements. The activity provides:

- Software Architectural and Detailed Design: Transforms the software requirements to the system software architecture and software de- tailed design.

- Software Construction: Develops the soft- ware code and data from the Software Design.

- Software Integration and Tests: Ensures that the integrated Software Components satisfy the software requirements.

- Product Delivery: Provides the integrated software product to the Customer.

As illustrated in figure 2, the customer's statement of work (SOW) is used to initiate the PM process. The project plan will be used to guide the execution of the software requirements analysis, software architectural and detailed design, software construction, and software integration and test, and product delivery activities. The PM process closure activity will deliver the Software Configuration (i.e. a set of software products such as documentation, code and tests) and will obtain the customer's acceptance to formalize the end of the project. 


\subsection{ISO/IEC 29110 Deployment Assistance}

A novel approach was taken to assist VSEs with the deployment of ISO/IEC 29110 and to provide guidance on the actual implementation this standard. A set of Deployment Packages (DPs) have been developed to define guidelines and explain in more detail the processes defined in the ISO/IEC 29110 profiles [26] A deployment package is not a complete process reference model. Deployment packages are not intended to preclude or discourage the use of additional guidelines that VSEs find useful. The elements of a typical DP are: description of processes, activities, tasks, steps, roles, products, templates, checklists, examples, references and mapping to standards and models, and a list of tools.

DPs were designed such that a VSE can implement its content, without having to implement the complete ISO/IEC 29110 framework, i.e. all the management and engineering activities, at the same time. A set of nine DPs have been developed and are freely available from [27].

\section{ISO/IEC 29110 INDUSTRY TRIAL}

In this section we will present 7 trial implementations of ISO/IEC 29110. The purpose of these trials is to illustrate the usage of this standard in an industrial context and to provide feedback to standards authors. Whilst not a detailed methodological approach to validation of this standard and whilst acknowledging the validation limitations, we believe that these high level results are useful to researchers and practitioners alike.

\subsection{Case 1: A Peruvian IT start-up}

Over $98 \%$ of Perú are micro, small and medium enterprises (MSMEs) having fewer than 10 workers. About 7,6 million people work in companies having fewer than 10 workers. About 14,000 Peruvian companies are associated with the Information Technology and Communications (ITC) industry [28].

An implementation of ISO/IEC 29110 has been conducted in a four-people start-up VSE created in 2012 [29]. During its two years of existence, the VSE has been involved in over 80 projects, most of which have lasted less than two months. The VSE used agile practices to implement software solutions such as Web 2.0 responsive design systems and mobile applications. After completing the implementation of the Basic profile of ISO/IEC 29110, the VSE executed in 2014 a project under contract. The product developed was a software solution that facilitates communication between clients and legal consultants at one of the largest insurance companies in Peru. The solution had to be implemented on a web platform and deployed into a cloud environment.

Since the VSE was using agile methods to implement its software projects, customer requirements were expressed as user stories. For this project, the VSE had determined that the duration of a sprint would be one week. The project had 6 sprints. All software components, test cases, test procedures and user stories were linked through a traceability matrix. As illustrated inn table 1, the total effort to implement 
the project was 882 hours. The effort devoted to prevention activities such as installation of the environment (servers, tools, etc.) was 14 hours, task execution took 585 hours, reviews took 124 hours and effort to correct defects identified in reviews and in testing took 159 hours. The start-up wasted only $18 \%$ of the total project effort (i.e. 159 hours/882 hours) on rework. Since it was the first time the VSE had executed the new ISO/IEC 29110 processes in a real project, so there was a learning curve that resulted in additional hours spent on rework for different project tasks. Despite this situation, the result was close to the percentage of rework (i.e. about $15 \%$ to $25 \%$ ) of an organization that has implemented the Capability Maturity Model and is at maturity level 3 .

Table 1. Effort to execute, detect and correct errors [29]

\begin{tabular}{|l|l|l|l|l|}
\hline Title of task & $\begin{array}{l}\text { Prevention } \\
\text { (hours) }\end{array}$ & $\begin{array}{l}\text { Execution } \\
\text { (Hours) }\end{array}$ & $\begin{array}{l}\text { Review } \\
\text { (Hours) }\end{array}$ & $\begin{array}{l}\text { Rework } \\
\text { (Hours) }\end{array}$ \\
\hline Environment installation & 14 & & & \\
\hline Project plan development & & 15 & 3 & 7 \\
\hline Plan execution, project assessment \& control & & 108 & & \\
\hline Specification development & & 107 & 28 & 58 \\
\hline Architecture development & & 35 & 10 & 14 \\
\hline Test plan development & & 45 & 8 & 11 \\
\hline Code development and testing & & 253 & 70 & 62 \\
\hline Develop user guide \& maintenance document & & 14 & 5 & 7 \\
\hline Product deployment & & 6 & & \\
\hline Project closure & & 2 & & \\
\hline Total hours & $\mathbf{1 4}$ & $\mathbf{5 8 5}$ & $\mathbf{1 2 4}$ & $\mathbf{1 5 9}$ \\
\hline
\end{tabular}

For the first stage of the audit process, the Peruvian VSE invested about 22 hours and $500 \$$ for the auditor. For the initial certification stage, the VSE invested about 63 hours. The cost of the auditor, excluding the travel expenses, was $1,500 \$$. The total effort and cost of an ISO/IEC 29110 audit is very small compared to a typical CMMI official assessment. This start-up became the first Peruvian VSE to obtain an ISO/IEC 29110 certification. The third stage of a certification cycle involves the completion of two surveillance audits one and two years after obtaining the initial certification. Finally, the fourth stage is the recertification of the VSE; once the 3-year certification cycle has elapsed.

In order to promote the recognition of qualifications between countries, there are international organizations such as the International Accreditation Forum (IAF). The IAF is the world association of conformity assessment accreditation bodies in the fields of management systems, products and services, and to date, it has more than 60 member countries. The Peruvian and the Brazilian accreditation bodies are members of this organization. An ISO/IEC 29110 certificate of conformity issued by an accreditation body member of the IAF is recognized by all members of IAF. The conformity certificate has become a major differentiator with regard to the main competitors of the VSE. The Peruvian start-up VSE has gained access to larger software develop- 
ment projects and increased its customer base. The VSE has increased its number of workers to date, from 4 to 10 employees.

\subsection{Case 2: A Canadian IT start-up}

An implementation project has been conducted in an IT start-up VSE by a team of two developers [25]. Their web application allows users to collaborate, share and plan their trips simply and accessible to all. The use of the Basic profile of ISO/IEC 29110 has guided the start-up to develop an application of high quality while using proven practices of ISO 29110 . The total effort of this project was nearly 1000 hours. The two members of the team were assigned roles and activities of ISO 29110. The management and engineering guide of the Basic profile lists the documents that have to be developed during a project as well as their typical content.

During the software development, a traceability matrix was developed between the software requirements, defined in the requirements specification document, and the software components. Since, in most projects requirements, defined in the requirements activity, are never finalized at the end of this activity, a traceability matrix is very useful. One advantage of such a matrix is the possibility of rapidly identifying the impacted software components when modifications, additions, deletions, of software requirements are done during a project.

Verification tasks, such as peer reviews, were performed on documents such as the requirement specifications and the architecture. The team used the desk-check to review their documents which is inexpensive and easy to implement in any organization and can be used to detect anomalies, omissions, improve a document or present and discuss alternative solutions.

As defined in ISO/IEC 29110, the software integration and tests activity ensures that the integrated Software Components satisfy the software requirements. This activity provides [30] work team review of the project plan to determine task assignment.

- Understanding of test cases and procedures and the integration environment.

- Integrated software components, corrected defects and documented results.

- Traceability of requirements and design to the integrated software product.

- Documented and verified operational and software user documentations.

- Verified software baseline.

To manage the defects detected, a tracking tool was used. Such software allowed the team to do an inventory of problems found during the integration and testing activity, to track problems and to classify them, and to determine a priority for each defect found. In this project, the open source Bugzilla software tool had been used to manage the defects.

The members of the start-up have recorded the effort, in person-hours, spent on tasks of the project to the nearest 30 minutes. For each major task, the effort to execute the task, the effort required to review a document, such as the software specification document, in order to detect errors and, the effort required to correct the errors (i.e. the rework). As an example, for the development of the software architecture 
document, it took 42.5 hours to develop, an additional 1.5-hour to conduct a review and an additional 3.5 hours to correct the errors.

For this start-up project, about $8.9 \%$ (i.e. 89 hours $/ 990.5$ hours) of the total project effort has been spent in prevention tasks such as the installation of the server, the workstations and the software tools; and only $12.6 \%$ has been spent on rework (i.e. 125 hours/990.5 hours). This indicates that the use of appropriate standards, in this case for a start-up company, can guide all the phases of the development of a product such that the wasted effort (i.e. rework) is about the same as a more mature organization (i.e. about level 3 of CMM).

In most start-ups, the wasted effort, for a project similar to this one, would have added about 90 hours (i.e. $30 \%$ of 716 or 215 hours -125 hours). This also implies, that for a net effort of about 6 hours per member per day (if we subtract from an 8hour day interruptions (e.g. phone call), answering emails, discussions in corridors, etc.), the product would have been ready for delivery to a customer about 15 days, of 6 hours, later than with a project with only $12.6 \%$ of waste.

These two projects have demonstrated that, by using ISO/IEC 29110, it was possible to properly plan the project and develop the software product using proven software practices documented in standards as well as not interfering with the creativity during the development of their web site. People who think that standards are a burden, an unnecessary overhead and a treat to creativity should look at this start-up project and revisit their results.

\subsection{Case 3: A Canadian/Tunisian IT start-up}

Metam is a company founded in 2013 by a software engineering graduate student of ÉTS. The company has one site in Canada and one site in Tunisia. Its business domains are software development services, web solutions, mobile applications as well as consulting services to implement ERP solutions. The Basic profile of ISO/IEC 29110 was used as the framework for the company's software processes. It was also used as a foundation to implement CMMI DEV level 2 practices because it was requested by some military contracts. In 2015, the VSE has 12 employees.

\subsection{Case 4: A large Canadian financial institution}

The Cash Management IT department, of a large Canadian financial institution, is responsible for the development and maintenance of software tools used by traders. The software team is composed of 6 people. Each year, the division is faced with an increase in the numbers of requests to add, correct or modify features related to supported applications. Before the implementation of the ISO 29110-agile process, customers had the following complaints:

- Very difficult to know the status of specific requests

- Very often, there is an incident when a change is put in production.

- There is a large number of faults detected by the quality assurance department 
- The development process is painful and the documentation produced is not very useful.

In response to this problem, we evaluated our process by comparing the activities of the maintenance process to those of the Basic profile of the ISO/IEC 29110. Some shortcomings were found in the project management process and in the software implementation process.

The project management process has been adapted to the context of the division, by injecting a few tasks of the SCRUM methodology. The new agile process, using the Basic profile of the ISO/IEC 29110, has been tested on three pilot projects. The new process helped to significantly reduce the number of major incidents caused by changes to the tools of the traders. The users are delighted with the new agile planning and control approach, which allows them to better manage their priorities and to always know the status of their requests. The maintenance team was also very pleased to see an improvement in the quality of the change requests, resulting in a noticeable decrease in the number of defects when handed to traders.

The adoption of this agile approach, however, requires a higher availability from the users. Initially, this new approach presented a challenge. In some cases, a few users appointed a representative to play the role of head of product backlog. But, that person did not have adequate knowledge of the business domain. Also, the head of product backlog was not able to respond quickly to questions from developers about the requirements, and user stories were not sufficiently documented in advance to maintain the velocity of the team. Finally, representatives of the Project Office and the Audit Group required a few modifications to the new ISO 29110-agile process.

A survey has been conducted to measure the satisfaction level of traders after the deployment of the new ISO 29110-agile process. The following ten questions were asked to traders (on a 0 to 10 scale):

- How do you qualify the quality of our software upgrades (e.g. number of incidents recorded in production)?

- Are you well informed about the content of the next software upgrade?

- Is the frequency of delivery right for you?

- How do you trust the new process?

- How would you describe the ability of the new process to respond to your needs?

- How easy is it to consult the status of a change request?

- How much the new process prioritizes the added value for you as a trader?

- What is the quality level of upgrades?

- Are you satisfied with the productivity of the team in response to your needs?

- What is your overall level of satisfaction about the new process (e.g. quality, cost, return on investment)?

The new ISO 29110-agile process has been tested on three pilot projects. The new process helped to significantly reduce the number of major incidents caused by changes to the tools of the traders. The users are delighted with the new agile planning and control approach, which allows them to better manage their priorities and to always know the status of their requests. The maintenance team was also very pleased 
to see an improvement in the quality of the change requests, resulting in a noticeable decrease in the number of defects in the software tools handed to traders.

\subsection{Case 5: A Canadian company in the automotive field}

TM4 is a Canadian company of more than 140 people, of whom 14 are directly employed as software engineers, the meeting the criteria of being a VSE. The company designs and sells electric powertrain systems in the automotive field. Their products are embedded software that controls the operation of engines in real time and software that controls the interactions between the components of a vehicle.

The company planned to increase its production systems in the coming years. Before this increase in production, and for the sake of improvement and compliance with standards, the company wanted to review and improve its software development processes.

The Basic Profile of ISO/IEC 29110 was used in this effort to improve its processes. A compliance study was conducted to establish the difference between the processes in place and those proposed by the ISO/IEC 29110. A pilot project has been successfully completed in May 2015. New software projects will use the ISO/IEC 29110-based processes.

An analysis of differences between ISO/IEC 29110 and ISO 26262, a standard for the automotive industry, was conducted and an economic impact assessment was conducted using the methodology developed by ISO [31].

\subsection{Case 6: A Canadian Transportation Enterprise}

A project was created to define and implement project management and engineering processes at CSinTrans Inc. (CSiT), a Canadian company, established in 2011 [32]. The company specializes in the integration of interactive systems, communication and security in the field of public transport such as trains, subways and buses and railway stations, and stations bus stops. Some customers in this domain are requiring from their suppliers to be assessed at CMMI Level 2. Implementing the practices of CMMI Level 2 was too demanding for a start-up. Instead, ISO/IEC 29110 standards and guides for systems engineering, developed from a subset of ISO/IEC/IEEE 15288 [53] and ISO/IEC/IEEE 15289 appropriate for VSEs, have been used as the main reference for the development of the processes of CSiT [37].

To avoid additional process and produce too many documents, participants gave themselves the 2 sets of guidelines:

- Regarding processes, the guideline was to add tasks not described in the Basic profile only if they add value to the context and projects of the company or provided an alignment with CMMI level 2.

- For the document templates, the guidelines were:

- Group different documents into one where this is possible;

- Each section of a template must be relevant and applicable. If a section does not provide added value, it is not included. 
The standard has helped raise the maturity of this young organization by implementing proven practices and developing uniform work products. ISO/IEC 29110 was a good starting point to align processes with selected level 2 and 3 practices of the CMMI model. Compliance with the ISO standard allowed CSiT to be recognized as producing quality products. ISO/IEC 29110 has also helped in developing lightweight processes allowing the small company to remain flexible as well as its ability to react quickly to its customers. CSiT performed an external audit of the management and engineering processes, mainly based on ISO 29110.

\subsection{Case 7: The Implementation in a division of an Engineering Enterprise}

A Canadian division of a large American engineering company, the Transmission \& Distribution of electricity division, has implemented a program to define and implement project management processes for their small-scale and medium-scale projects [51. The firm already had a robust and proven process to manage their largescale projects. The objectives of this process improvement project were to reduce cost overruns and project delays, standardize practices to facilitate the integration of new managers, increase the level of customer satisfaction and to reduce risk-related planning deviations. Their projects are classified into three categories as illustrated in Table 2. As illustrated in the table, over $95 \%$ of the projects fall in the small- and medium-scale categories.

Table 2. Classification of projects by the engineering firm [51]

\begin{tabular}{|l|c|c|c|}
\hline & Small project & Medium project & Large project \\
\hline Duration & $<2$ months & $>2$ and $<8$ months & $>8$ months \\
\hline Team size & $<=4$ people & $4-8$ people & $>8$ people \\
\hline No. of engineering specialties & 1 & $>1$ & Many \\
\hline Engineering fees & $\$ 5,000-\$ 70,000$ & $\$ 50,000-\$ 350,000$ & $>\$ 350,000$ \\
\hline Percentage of projects & $70 \%$ & $25 \%$ & $5 \%$ \\
\hline
\end{tabular}

Pilot projects have been conducted to test the project management processes and associated support tools (e.g. templates, checklists). The pilot projects consisted of running three different projects where project managers implemented the process and the associated tools. Managers then evaluated the proposed processes, identified problems and potential improvements.

The project management practices used by the company's managers were assessed against the ISO standard's Basic Profile. The division used the project management process of the Entry Profile of ISO 29110 to document their small-scale project management process and they used the project management process of the Basic profile to document their medium-scale project management process.

Pilot projects have been conducted to test the project management processes and associated support tools (e.g. templates, checklists). The pilot projects consisted of running three different projects where project managers implemented the process and 
the associated tools. Managers then evaluated the proposed processes, identified problems and potential improvements. The lessons learned sessions conducted at the end of the pilot projects have identified minor adjustments to the processes and tools.

A section of the intranet, dedicated to project management, was created and served as a main access to project management documents such as project management process guides, checklists, forms and templates. Project managers were trained in the new processes and support tools.

The tools developed to support the project management processes proved very useful and helped the project managers rapidly integrate the knowledge required to execute the processes. The improvement program was so successful that managers of the company's other divisions have shown an interest in learning this approach in order to implement it within their respective divisions.

ISO has developed a methodology to assess and communicate the economic benefits of standards (ISO 2010), which was used, by the engineering firm, to estimate the anticipated costs and benefits over a period of three years. The key objectives of the ISO methodology are to provide:

The sponsors of this process definition project made the estimates. The improvement program project sponsors made an estimate of anticipated costs and benefits over a period of three years. Table 3 shows the results for the first three years.

Table 3. Costs and benefits estimations [51]

\begin{tabular}{|l|c|c|c|c|}
\hline & Year 1 & Year 2 & Year 3 & Total \\
\hline $\begin{array}{l}\text { Implement } \\
\text { \& maintain }\end{array}$ & $59600 \$$ & $50100 \$$ & $50100 \$$ & $159800 \$$ \\
\hline Net Benefits & $255500 \$$ & $265000 \$$ & $265000 \$$ & $785500 \$$ \\
\hline
\end{tabular}

The engineering firm is planning to document and implement their systems engineering processes for the small-scale and medium scale projects using the Entry and Basic Profiles of the ISO 29110 systems engineering standard and guides.

\section{DISCUSSION AND FUTURE WORK}

This section will present some discussion on the pilot case study implementation and well as describe future work in relation for the continued development of ISO/IEC 29110 set of standards.

\subsection{Discussion}

The seven pilot case studies presented in this paper have demonstrated that by using ISO/IEC 29110, it was possible to properly plan and execute projects and develop products or conduct projects using proven system or software engineering practices without interfering with the creativity of developers. The relationship between the success of a software company and the software process it utilized has been investi- 
gated $[33,34]$ showing the need for all organizations, not just VSEs to pay attention to software process practices such as ISO standards.

\subsection{Planned Standard Development}

As ISO/IEC 29110 is an emerging standard there is much work yet to be completed. The main remaining work item is to finalize the development of the remaining two software profiles of the Generic Profile Group: (a) Intermediate - management of more than one project and (b) Advanced - business management and portfolio management practices.

Working Group 24 of ISO/IEC JTC1/SC7 who were initially authorized to develop the ISO/IEC 29110 for software, was also assigned to develop a similar approach for VSEs involved in the domain of systems engineering $[35,36]$. Recently the ISO published the systems engineering and management guide of the Basic profile ISO/IEC TR 29110-5-6-2:2014 [37] and Entry ISO/IEC TR 29110-5-6-1:2015 [38].

Work currently underway on an assessment mechanism for ISO/IEC 29110 [39], a clear niche market need is emerging which may force the process assessment community to change their views on how process assessments are carried out for VSEs. It is clear that the process assessment community will have to rethink process assessment, new methods and ideas for assessing processes in VSEs.

\subsection{Standards Education}

In 2009, it was proposed to establish an informal interest group about education. Its main objective is to develop a set of courses for software undergraduate and graduate students such that students learn about the ISO standards for Very Small Entities (VSEs) before they graduate.

One way to develop standards professionals is by having professional graduate students involved in the application and improvement of international standards. At the Ecole de technologie superieure (ETS), a 7,800-student engineering school of Montréal, International Software Engineering Standards are introduced and used in Software Quality Assurance and Software Process Improvement courses and industrial projects conducted by graduate professional software engineering and IT students [55].

The role of education $[45,46,47]$ is a significant issue in ensuring that the next generation of software project managers and software process engineers are both familiar with the benefits of standards, specifically in VSEs and the role of ISO/IEC 29110 in particular. Such education programmes may assist with addressing the perceived issues with standards adoption and the lack of managerial commitment [48, 49] in adopting VSE standards.

\section{ADDITIONAL INFORMATION}

The following web site provides more information about ISO/IEC 29110: 
http://profs.logti.etsmtl.ca/claporte/English/VSE/index.html

\section{REFERENCES}

1. Coleman, G., \& O'Connor, R. V. (2008). An investigation into software development process formation in software start-ups. Journal of Enterprise Information Management, 21(6), 633-648.

2. O'Connor, R. V., \& Laporte, C. Y., 2012. Software project management in very small entities with ISO/IEC 29110 (pp. 330-341). Springer Berlin Heidelberg

3. Basri, S., \& O'Connor, R. V. 2010. Evaluation on knowledge management process in very small software companies: a survey, 5th Knowledge Management International Conference, Terengganu, Malaysia, May 2010.

4. Mora, M., O'Connor, R., Raisinghani, M., \& Macías-Luévano, J. 2011. An IT service engineering and management framework (ITS-EMF). International Journal of Service Science, Management, Engineering, and Technology, 2(2), 1-15.

5. Laporte, C.Y., Alexandre, S., and O'Connor, R. 2008. A Software Engineering Lifecycle Standard for Very Small Enterprises, R.V. O'Connor et al (Eds) Proceedings of EuroSPI Springer-Verlag, CCIS Vol. 16, pp. 129-141,

6. Statistics Canada. 2008. Available at: http://www.ic.gc.ca/sbstatistics

7. O'Connor R. and Laporte, C.Y., 2010. Towards the provision of assistance for very small entities in deploying software lifecycle standards. In Proceedings of the 11th International Conference on Product Focused Software (PROFES '10). ACM.

8. O'Connor R. and Coleman G., 2009. Ignoring 'Best Practice': Why Irish Software SMEs are rejecting CMMI and ISO 9000, Australasian Journal of Information Systems, Vol. 16, No. 1 ,

9. Basri S, O’Connor RV. 2011 A study of software development team dynamics in SPI. Systems, Software and Services Process Improvement (EuroSPI 2011), CCIS, vol. 172, pp. 143-154. Springer-Verlag.

10. Coleman, G., O'Connor, R. 2006. Software Process in Practice: A Grounded Theory of the Irish Software Industry. In: Richardson, I., Runeson, P., Messnarz, R. (eds.) EuroSPI 2006. LNCS, vol. 4257, pp. 28-39. Springer, Heidelberg.

11. Petkov, D., Edgar-Nevill, D., Madachy, R., \& O'Connor, R. 2008. Information systems, software engineering, and systems thinking: Challenges and opportunities. International Journal of Information Technologies and Systems Approach (IJITSA), 1(1), 62-78.

12. O'Connor, R., \& Basri, S., 2012. The effect of team dynamics on software development process improvement. International Journal of Human Capital and Information Technology Professionals, 3(3), 13-26.

13. O'Connor, R. V., \& Coleman, G. 2007. An investigation of barriers to the adoption of software process best practice models. ACIS 2007 Proceedings, 35.

14. O'Connor, R. and Laporte, C.Y., 2011 Deploying Lifecycle profiles for Very Small Entities: An Early Stage Industry View, Proceedings of 11th International SPICE Conference on Process Improvement and Capability dEtermination, CCIS Vol. 155, Springer-Verlag, May 2011.

15. O'Connor, R. and Laporte, C.Y., 2011b. Using ISO/IEC 29110 to Harness Process Improvement in Very Small Entities, Workshop on SPI in SMEs, 18th European Software Process Improvement Conference, CCIS Vol. 172, Springer-Verlag. 
16. Laporte, C.Y., O'Connor, R., Fanmuy, G., 2013. International Systems and Software Engineering Standards for Very Small Entities, CrossTalk - The Journal of Defense Software Engineering, May/June 2013, Vol. 26, No 3, pp 28-33.

17. Laporte, C.Y., Séguin, N., Villas Boas, G., 2013b. Seizing the benefits of software and systems engineering standards, ISO Focus + , International Organization for Standardization, February 2013, pp 32-36.

18. O'Connor, R. 2012. Evaluating Management Sentiment Towards ISO/IEC 29110 in Very Small Software Development Companies. In: Mas, et al. (eds.) Software Process Improvement and Capability Determination. CCIS, vol. 290, pp. 277-281. Springer-Verlag, Heidelberg.

19. Ribaud, V., Saliou, P., O'Connor, R., Laporte, C.Y.: 2010. Software Engineering Support Activities for Very Small Entities. In: Riel, et al. (eds.) Systems, Software and Services Process Improvement. CCIS, vol. 99, pp. 165-176. Springer-Verlag, Heidelberg.

20. Galvan, S., Mora, M., O'Connor, R. V., Acosta, F., \& Alvarez, F. (2015). A Compliance Analysis of Agile Methodologies with the ISO/IEC 29110 Project Management Process. Procedia Computer Science, 64, 188-195.

21. Clarke, P., O'Connor, R.V. 2011. The Meaning of Success for Software SMEs: An Holistic Scorecard Based Approach. Systems, Software and Services Process Improvement (EuroSPI 2011), CCIS, vol. 172, pp. 272-83. Springer-Verlag, Heidelberg.

22. O'Connor, Rory and Paul, Clarke (2015) Software process reflexivity and business performance: initial results from an empirical study. In: International Conference on Software and System Process (ICSSP 2015), 24-26 Aug 2015

23. Jeners, S., Clarke, P., O'Connor, R. V., Buglione, L., and Lepmets, M. Harmonizing Software Development Processes with Software Development Settings - A Systematic Approach, In McCafery, F., O'Connor, R.V. and Messnarz R. (Eds), Systems, Software and Services Process Improvement, CCIS 364, Springer-Verlag, 2013

24. Clarke, P. and O'Connor, R., The situational factors that affect the software development process: Towards a comprehensive reference framework, Journal of Information and Software Technology, Vol. 54, Issue 5, May 2012. pp. 433-447

25. Laporte, C.Y., Hébert, C., Mineau, C., 2014. Development of a Social Network Website Using the New ISO/IEC 29110 Standard Developed Specifically for Very Small Entities, Software Quality Professional Journal, ASQ, vol. 16, no. 4, pp 4-25, 2014

26. O'Connor, R. V. and Laporte, C. Y., 2014. An Innovative Approach to the Development of an International Software Process Lifecycle Standard for Very Small Entities, International Journal of Information Technology and the Systems Approach, Vol. 7, No. 1, pp. 1-22.

27. Deployment Packages repository, [online] available from: http://profs.logti.etsmtl.ca/claporte/English/VSE/index.html

28. Krasner, H. 1998. Using the cost of quality approach for software. Crosstalk - The Journal of Defense Software Engineering 11 (November):6-11

29. Garcia, L., Laporte, C.Y., Arteaga, J., Bruggmann, M., Implementation and Certification of ISO/IEC 29110 in an IT Startup in Peru, Software Quality Professional Journal, ASQ, vol. 17, no. 2, 2015, pp 16-29.

30. ISO/IEC TR 29110-1:2011, "Software Engineering - Lifecycle Profiles for Very Small Entities (VSEs) - Part 1: Overview”. Geneva: International Organization for Standardization (ISO), 2011. Available at no cost from ISO at: http://standards.iso.org/ittf/PubliclyAvailableStandards/c051150_ISO_IEC_TR_291101 2011.zip

31. ISSO, Economic Benefits of Standards, Methodology guide» Version 2.0, 2013. International Organization for Standardization, Geneva, Switzerland 
32. Tremblay, N., Menaceur, J., Poliquin, D., Laporte, C.Y., Mise en place de processus de gestion de projets et d'ingénierie système chez CSiT, une entreprise canadienne dans le domaine du transport collectif, Revue Génie Logiciel, Numéro 114, Septembre 2015, pp 11- 27.

33. Laporte, C. Y., O'Connor, R. V. 2014. A Systems Process Lifecycle Standard for Very Small Entities: Development and Pilot Trials, In Barafort, B., O'Connor, R.V. and Messnarz R. (Eds), Systems, Software and Services Process Improvement, CCIS Vol. 425, Springer-Verlag.

34. O'Connor, R. V., and Basri, S. 2014. Understanding the role of knowledge management in software development: a case study in very small companies, International Journal of Systems and Service-Oriented Engineering, Vol. 4, No. 1, pp. 39-52.

35. Laporte, C.Y.; O'Connor, Rory V. 2014. Systems and Software Engineering Standards for Very Small Entities: Implementation and Initial Results. 9th International Conference on the Quality of Information and Communications Technology (QUATIC), pp.38-47, 23-26 Sept.

36. O'Connor, R. V., \& Sanders, M. 2013. Lessons from a pilot implementation of ISO/IEC 29110 in a group of very small Irish companies. In Software Process Improvement and Capability Determination (pp. 243-246). Springer Berlin Heidelberg.

37. ISO/IEC TR 29110-5-6-2:2014 - Systems Engineering - Lifecycle Profiles for Very Small Entities (VSEs) - Part 5-6-2: Systems engineering - Management and engineering guide: Generic profile group: Basic profile, International Organization for Standardization/International Electrotechnical Commission: Geneva, Switzerland. Available at no cost from ISO

http://standards.iso.org/ittf/PubliclyAvailableStandards/c063371_ISO_IEC_29110-56_2_2014.zip

38. ISO/IEC TR 29110-5-6-1:2015 - Systems and software engineering - Lifecycle Profiles for Very Small Entities (VSEs) -Part 5-6-1: System engineering Management and engineering guide: Generic profile group: Entry profile, International Organization for Standardization/International Electrotechnical Commission: Geneva, Switzerland. Available at no cost from ISO at: http://standards.iso.org/ittf/PubliclyAvailableStandards/index.html

39. ISO/IEC 29110-4-1:2011, Software Engineering -- Lifecycle Profiles for Very Small Entities (VSEs) - Part 4-1: Profile specifications: Generic profile group. Geneva: International Organization for Standardization (ISO), 2011.

40. ISO/IEC/IEEE 12207, Systems and software engineering- Software life cycle processes. International Organization for Standardization/International Electrotechnical Commission: Geneva, Switzerland.

41. Clarke, P., \& O'Connor, R. 2010. Harnessing ISO/IEC 12207 to Examine the Extent of SPI Activity in an Organisation. In Systems, Software and Services Process Improvement (pp. 25-36). Springer Berlin Heidelberg.

42. ISO/IEC/IEEE 15289, Systems and software engineering - Content of systems and software life cycle process information products (Documentation), International Organization for Standardization/International Electrotechnical Commission: Geneva, Switzerland

43. O'Connor, R., Basri, S. and Coleman, G., 2010. Exploring Managerial Commitment towards SPI in Small and Very Small Enterprises, in Riel et al (Eds), Systems, Software and Services Process Improvement, CCIS Vol. 99, Springer-Verlag, pp. 268-278.

44. Basri, S., \& O'Connor, R. (2012). A study of knowledge management process practices in very small software companies. American Journal of Economics and Business Administration, 3(4), 636-644 
45. Laporte, C.Y., O'Connor, R, Software Process Improvement in Graduate Software Engineering Programs, in: O'Connor, R.V., Mitasiunas, A. and Ross, M. (Eds), Proceeding of the 1st International Workshop on Software Process Education, Training and Professionalism (SPETP 2015), CEUR Electronic Workshop Proceedings, Vol. 1368, 2015, pp. 18-24.

46. Laporte, C.Y. and O'Connor, R.V., Software Process Improvement in Graduate Software Engineering Programs, proceedings 1st International Workshop Software Process Education, Training and Professionalism (SPEPT 2015), pp. 18 - 24, CEUR Workshop Proceedings, 2015.

47. Ribaud, V., Matthieu, A. B. and O'Connor, R.V., Process Assessment Issues in a Bachelor Capstone Project, proceedings 1st International Workshop Software Process Education, Training and Professionalism (SPEPT 2015), pp. 25 - 33, CEUR Workshop Proceedings, 2015

48. Sanchez-Gordon, M.L., O’Connor R.V. and Colomo-Palacios, R., Evaluating VSEs Viewpoint and Sentiment Towards the ISO/IEC 29110 Standard: A Two Country Grounded Theory Study, In Rout, T. and O'Connor, R.V. and Dorling, A. (Eds), Software Process Improvement and Capability Determination, CCIS 526, Springer-Verlag, 2015.

49. Basri, S. and O'Connor, R., Organizational Commitment Towards Software Process Improvement An Irish Software VSEs Case Study, 4th International Symposium on Information Technology 2010 (ITSim 2010), Kuala Lumpur, Malaysia, June 2010.

50. Ribaud, V. and O'Connor, R.V., Towards a blended Assessment Model for Small Entities, In O'Connor, R.V. Akkaya, M., Kemaneci K., Yilmaz, M., Poth, A. and Messnarz R. (Eds), Systems, Software and Services Process Improvement, CCIS 543, Springer-Verlag, 2015.

51. Laporte, C.Y., Chevalier, F., An Innovative Approach to the Development of Project Management Processes for Small-scale Projects in a large Engineering Company, 25th Annual International Symposium of INCOSE (International Council on Systems Engineering), Seattle, US, July 13-16, 2015.

52. ISO/IEC TR 29110-5-1-1:2011 - Software engineering - Lifecycle Profiles for Very Small Entities (VSEs) -Part 5-2-1: Management and engineering guide: Generic profile group: Entry profile, International Organization for Standardization/International Electrotechnical Commission: Geneva, Switzerland. Available at no cost from ISO at: http://standards.iso.org/ittf/PubliclyAvailableStandards/c051153_ISO_IEC_TR_29110-51_2011.zip

53. ISO/IEC/IEEE 15288, Systems and software engineering- System life cycle processes. International Organization for Standardization/International Electrotechnical Commission: Geneva, Switzerland.

54. Clarke, P., \& O'Connor, R. V. (2011). An approach to evaluating software process adaptation. In Software Process Improvement and Capability Determination (pp. 28-41). Springer Berlin Heidelberg.

55. Laporte, C., O'Connor, R., Garcia Paucar, L., \& Gerancon, B. (2015). An innovative approach in developing standard professionals by involving software engineering students in implementing and improving international standards. Standards Engineering: The Journal of SES (The Society for Standards Professionals), 67(2), 2-9. 